\title{
Identification of Risk Factors for Falls in the Elderly
}

\author{
Suzi Rosa Miziara Soares ${ }^{1}$, Alcimar Barbosa Soares ${ }^{2}$ and Angela Abreu Rosa de Sá ${ }^{2}$ \\ 1. Departament of Physioterapy, University for the Development of the State and of the Pantanal Region, Campo Grande 79070-900, \\ Mato Grosso do Sul, Brazil
}

2. Biomedical Engineering Laboratory, Electrical Engineering Faculty, Federal University of Uberlândia, Uberlândia 38408-100, Minas Gerais, Brazil

Received: September 22, 2013/ Accepted: October 26, 2013 / Published: December 31, 2013.

\begin{abstract}
Several technological apparatus and functional clinical trials are developed for the evaluation of the fall in the elderly. However, it is noted that these in their vast majority, evaluate the population generally, disregarding gender or age. In the research, the sample was stratified into: FG (Female Group); MG (Male Group); aged 60 to 69 years old (FG1 and MG1); aged 70 years old (FG2 and MG2). During the evaluation of the sample in each group, it was verified the amount of the falls, Med (medication/day), BMI (body mass index), CC (the circumference of the calf), CW (circumference of the waist), HGS (hand grip strength), gait (Dynamic Gait index-DGI), balance (Berg Balance Scale-BBS), mobility (test stand and walk timed-TUG), cognitive state (mini-mental state examination-MMSE), life satisfaction (Satisfaction with Life Scale—SLS), physical self-efficacy (Physical Evaluation Scale—PES), FC (functional capacity) and fear of falling (International Scale Effectiveness of falls_-FES-I-Brazil). In results, the falls, in each group, were: (1) FG: 53\%; (2) MG: 14\%; (3) FG1: 39\%; (4) FG2: 62\%; (5) MG1: 6\%; (6) MG2: 27\%. The Spearman's test ( $p<0.05$ ) established a correlation between falls, risk factors and clinical tests. In each group, were significant the following risk factors: (1) on FG: Med, CW, HGS, DGI, FES-I-Brazil, TUG, SLS, FC; (2) In FG2: DGI; (3) In MG: TUG; (4) In MG2: FES-I-Brazil ( $p<0.00$ ). The identification of clinical tests and risk factors of falling in elderly, distinguished by gender and age, clearly demonstrate that the assessment protocols shall be prepared differently. Thus, it was possible to establish new strategies to contribute to reducing the incidence of falls.
\end{abstract}

Key words: Elderly, fall, age, clinical protocols.

\section{Introduction}

Aging is a physiological process, progressive and dynamic, which records a series of instabilities and changes, not uniforms, in all systems of the body, that decrease efficiency in PDLA (performances of daily life activities) and in the maintenance of body posture $[1,2]$. These changes, in some aspects, may seem small and insignificant, however, the sum of these deficits increases the risk of incorrect or inefficient answers, with loss of coordination and instabilities, that can result in falls [3].

The falls have been seen as an epidemic in the third age, and as the most important factor of change in life of

Corresponding author: Suzi Rosa Miziara Soares, Ph.D., professor, research field: physical therapy. E-mail: srmiziara@gmail.com. elderly, because they indicate the beginning of body functional decline and may also be indicative of grievance or the emergence of a new medical condition. Its consequences are reflected in the physical aspect generating losses that compromise the musculoskeletal system [4]; in the emotional aspect (low self-esteem, depression, dependency, etc.); in the socioeconomic aspect (high costs for the family of the elderly) and for the health systems due to hospitalizations, extensive periods of rehabilitation and subsequent dependency $[1,2]$.

Faced with this problem and of the various causes of the problem, the global scientific community has researched, incessantly, to find more effective measures for the identification of the elderly with risk of falls and/or recurrent falls. Even the elderly healthy and active, can suffer falls in various situations, as a 
result of distractions or momentary risk behaviors. Several technological apparatus and functional clinical trials are developed for the evaluation of the fall in the elderly, and can be used individually or jointly in the form of evaluation protocols. However, it is noted that these in their vast majority, evaluate the population generally, disregarding gender or age.

Considering the morphofunctional changes different between genders, age groups and the high rates of fall, this study is aim to verify if the assessment protocols for predicting falls in the elderly would be most predictive if they were laminated differently by gender and age. And also, it will be verified if those protocols should be developed with specific techniques for each of the laminates.

The question is not contest the veracity of the protocols and evaluation procedures used so far, but attempting to set the evaluation techniques reflecting more effectively the real condition of each individual. Thus, the detection and prediction of subsequent outcomes could be more efficient, allowing the doctor to make decisions, and carry out clinical interventions more effective, to prevent future adverse events (falls), aiming at the maintenance or improvement of the quality of life of the elderly.

\section{Materials and Methods}

\subsection{Location and Research Subjects}

The study was carried out at Senior Center Elias Lahdo (Campo Grande/MS, Brazil). The methodological procedures were reviewed and approved by the Committee of Ethics in Research with Human Beings at the Federal University of Uberlândia-CEPUFU (Protocol nr. 21806).

For the study, 85 individuals over the age of 60 years old, of both sexes, not institutionalized, were selected. They were regular physical activity practitioners (minimum of one year), who showed no medical weaknesses when undertaking the balance. The individuals selected signed an informed consent, for voluntary participation in the study.

\subsection{Procedures and Research Tools}

It has elaborated a protocol, which consisted of anthropometric measurements: weight, height, BMI (body mass index), CC (circumference of the calf), CW (circumference of the waist) and the HGS (hand grip strength) quantified by Jamar® dynamometer in member of motor dominance, indicated by the volunteer. The protocol was composed also by scales and functional tests to assess the physical and emotional status of volunteers. The scales and selected tests to evaluate the physical status were the DGI (dynamic gait index), BBS (berg balance scale) and TUG (test of timed up and go). The cognitive state was evaluated through MMSE (mini-mental state examination). The emotional status was assessed through SLS (satisfaction with life scale), SES (self-efficacy scale), multidimensional scale of FC (functional capacity) and FES-I-Brazil (international scale effectiveness of falls).

This protocol was chosen by contemplating the more used tests in physiotherapy. Thus, the advantage of the use of these methods is to be possible to repeat the methodology presented by any healthcare professional. And to identify and analyze the variables that were collected in this protocol has been used the Matlab software ${ }^{\circledR}$. The advantage of using it is that it includes a large set of statistical packages and allows the user to do the necessary programming, through the selection of data, and extract the data of interest.

\section{Results}

The 85 volunteers evaluated ( 28 men and 57 women) were stratified by sex, FG (Female Group) and MG (Male Group), and by age groups: FG1 and MG1 (60 to 69 years old) and FG2 and MG2 (over 70 years old). The groups were composed as follows:

MG1: 17 individuals (20\%);

MG2: 11 individuals (12.94\%);

FG1: 23 individuals (27.1\%);

FG2: 34 individuals (40\%).

With respect to the fall, the maximum reported falls 
was five in the last 12 months and $40 \%$ of the sample reported at least one fall in this period. With respect to fall by sex, it was verified that the FG falls more (53\%) than MG (14\%).

And with respect to fall by age, the FG2 falls more than other groups (62\%) followed by FG1 (39\%), MG2 (27\%) and MG1 (6\%). Risk factors that presented a correlation with fall in MG were BBS (0.0272) and TUG (0.0230). In MG2, FES-I-Brazil and MG1 (both with correlation $<0.0001$ ), due to only one volunteer reported fall, was not observed any significant correlation. In FG the factors were: Med (0.0009), CW (0.0172), HGS (0.0214), DGI (0.0015), FES-I-Brazil (0.0001), TUG (0.0054), SLS (0.0488) and FC (0.0169).

In FG1 was not observed a significant correlation and in the group FG2, the DGI was 0.0316 .

In order to identify which risk factors (variables) that contributed with the indexes of falls, a computational program has been developed using the software Matlab $^{\circledR}$, to calculate the covariance of all groups in relation to fall. Thus, the following operations were performed: the calculation of the mean and standard deviation of each factor. Then, these data were normalized by subtracting each sample of the variable by the mean of population, and then dividing by the standard deviation. Finally, it has been estimated the covariance of the normalized data. To calculate the correlation was used only presence or absence of fall of the patients who participated in the research. As the covariances have positive or negative values, they were compared in terms of their absolute values ("strength" of the relationship). As a criterion of comparison was established that those values highlight should be above average in 1 (one) standard deviation. So, if the value of the covariance is positive, the risk factor and the fall ranged in the same direction; increasing or decreasing its indexes. On the other hand, if the value of the covariance presented negative result, the risk factor and the fall index varied in opposite directions. Figs. 1-3 illustrate the laminate results of covariances by groups of samples, male and female group, respectively.

The average of covariances in this group was $0.26 \pm$ 0.16 and the cut-off point was 0.42 . Thus, the factors that presented covariance above the cut-off point were considered to be heavily dependent on variations in fall. They are: Med (0.47); HGS (0.43); BMI (0.51); Fes-I-Brazil (0.48) and TUG (0.45). In relation to values obtained, the Med, Fes-I-Brazil and TUG

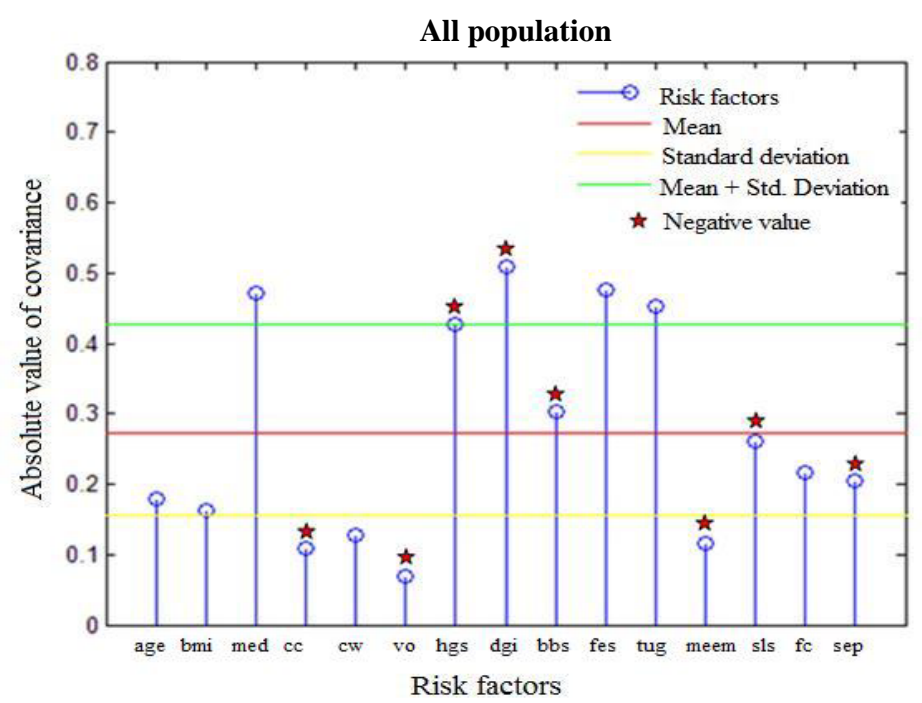

Fig. 1 Covariance indexes (absolute values) of the risk factors and clinical trials with falls across the sample.

age = age; bmi = body mass index; med = medication; $\mathrm{cc}=$ circumference of the calf; $\mathrm{cw}=$ circumference of the waist; vo = visual orthosis; hgs = hand grip strength; dgi = dynamic gait index; bbs = berg balance scale; fes =Fes-I-Brasilr; tug = timed up and go; meem $=$ mini-mental Test; $\mathrm{sls}=$ satisfaction with life scale; $\mathrm{fc}=$ multidimensional assessment of functional capacity; sep = self-efficacy scale physics. 


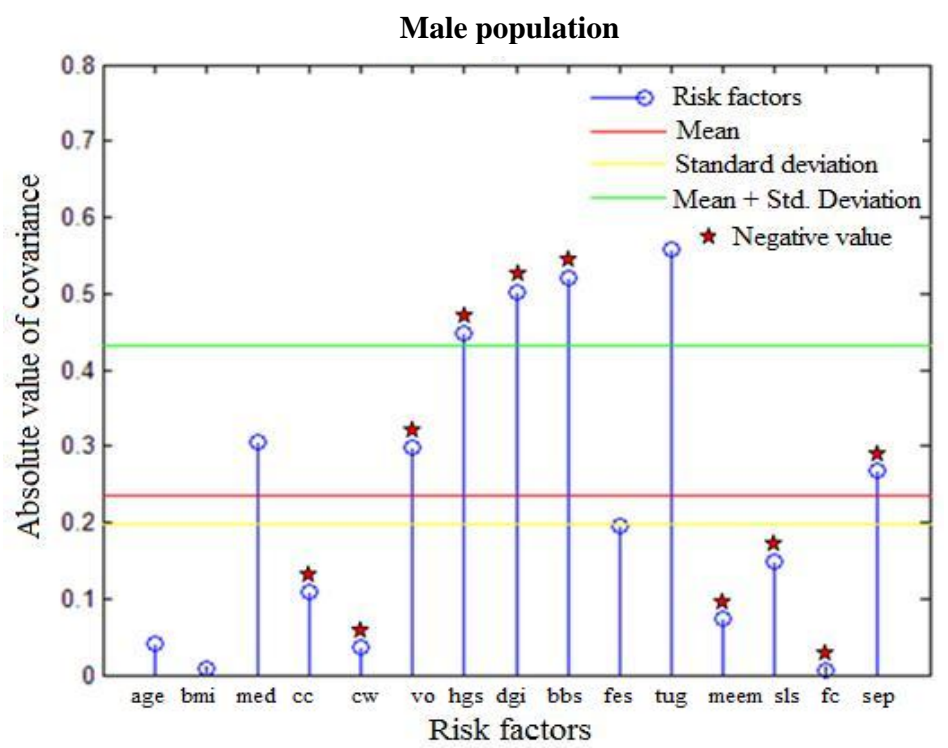

Fig. 2 Covariance indexes (absolute values) of the risk factors and clinical trials with falls, in the male sample-MG group.

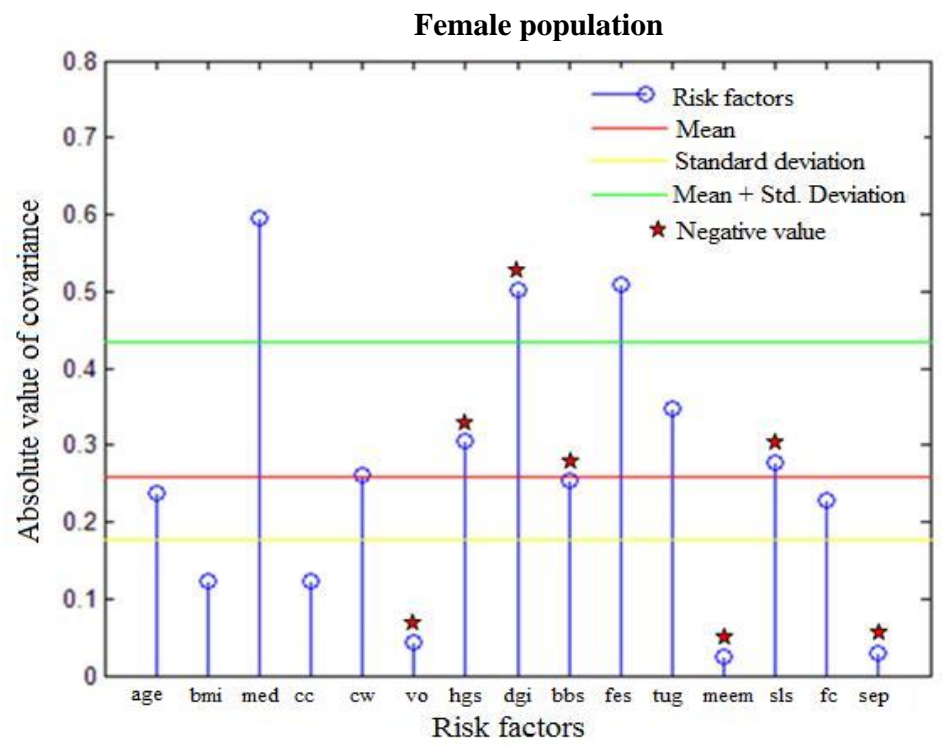

Fig. 3 Covariance indexes (absolute values) of the risk factors and clinical trials with falls, in the female sample-FG group.

presented positive covariance, indicate a variation with fall, i.e., increase or decrease their scores in relation to the quantity of falls. The HGS and DGI showed negative covariance, indicating that the higher the HGS or the score on DGI, the lower the index fall, or vice versa.

The average of covariances in the male group was $0.22 \pm 0.20$ and the cut-off point was 0.42 ; and the indexes that achieved prominence were: HGS (0.47), BMI (0.50), BBS (0.52) and TUG (0.56). All of these factors, with the exception of the TUG, had negative covariance. In the group MG1, the cut-off point was 0.51 ; and the indexes that achieved prominence were: HGS (0.53), DGI (0.72) and TUG (0.78). HGS and DGI showed negative covariance, and the TUG positive correlation. In the group MG2, the average of covariances was $0.29 \pm 0.19$ and the cut-off point was 0.47. Prominence: BBS (0.67) with negative covariance and FES-I-Brazil (0.53) with positive covariance.

In FG (Female Group), the average was $0.25 \pm 0.17$ and the cut-off point was 0.42 . Prominence: Med (0.59) 
and FES-I-Brazil (0.51) with positive covariance, indicating that these factors and falls, vary with falls. The DGI (0.50) was also highlighted, however, showed negative correlation, i.e., it ranges from inverse way in relation to fall. Through stratification by age in the FG1 the mean was $0.25 \pm 0.16$, and the cut-off point was 0.42. In this group, the following indices were differentiated: BMI (0.43), CW (0.44), FES-I-Brazil (0.53) and TUG (0.42) -all showed positive covariance. In FG2 group, the average was $0.29 \pm 0.19$ and the cut-off point was 0.48 . The risk factors with covariance above this value may be considered strongly dependent on variations of falls, they are: Med (0.65), CC (0.54)-positive covariance; and DGI (0.56) —negative covariance.

The results shown in Table 1 relate to the risk factors and falls within the laminate groups in this study.

It is clear that the risk factors are distinguished by gender and age group and should be considered in evaluation protocols and intervention programmes falling in elderly.

Table 1 Results of the relevance of risk factors by sex and age group.

\begin{tabular}{|c|c|c|c|c|c|c|}
\hline Risk factor risco & $\mathrm{FG}$ & FG1 & FG2 & MG & MG1 & MG2 \\
\hline AGE & & & & & & \\
\hline BMI & & & & & & \\
\hline OrtV & & & & & & \\
\hline MED & & & & & & \\
\hline $\mathrm{CC}$ & & & & & & \\
\hline $\mathrm{CW}$ & & & & & & \\
\hline HGS & & & & & & \\
\hline DGI & & & & & & \\
\hline BBS & & & & & & \\
\hline TUG & & & & & & \\
\hline MMSE & & & & & & \\
\hline PES & & & & & & \\
\hline $\mathrm{FC}$ & & & & & & \\
\hline SLS & & & & & & \\
\hline FES-I-Brazil & & & & & & \\
\hline
\end{tabular}

\section{Discussion}

The study was considered only health patients and that used to practice some regular physical activity, at least for one year. This way, no one of them experienced falls which led to hospitalization or death.

The registered indexes falls in the elderly population continue growing, because its proportion has been regarded as a public health problem that affects the global scientific community. The multifactorial nature of the event indicates the difficulty in identifying the predictors of fall, what makes difficult interventions before the occurrence of the event.

Technological apparatus with high content added as well as scales and clinical trials, have been developed by the scientific community in an attempt to predict the fall. However, these have not proved effective in solving the problem that has become a global challenge. The assessment procedures are applied in global population and are widespread, not taking into consideration the gender or age of the individual. Also there are interventions that support physical activity programs that are effective for improving life quality maintenance. However, they are not sufficient to prevent falls, because even during the practice of physical activity, the individual may fall $[5,6]$.

So to compose the evaluation protocol of this study, were selected scales and functional tests to cover the individual, both physical and emotional status, because only one measuring instrument does not translate the real condition of functioning of the organ systems, what prevents the identification of the factors that trigger or that deserve special attention in the prediction of falling in elderly $[1,4,7]$.

This same procedure was performed by Boulgarides [8], which evaluated 99 elderly, with an average of 74 years old, living in community, using clinical instrument as a platform of strength, clinical trials (BBS, TUG and DGI), and also analyzed some health factors (physical activity level) and socio-demographic. They followed the history of falls during one year, and concluded that the simultaneous knowledge of 
cognitive changes and posture can better predict falls, when compared to the isolated testing that consider only the postural control components.

Reaffirming the necessity of multifactorial evaluations, Sjösten [5], conducted a randomized study (during 2003-2005), with 293 individuals, with an average age of 73.5 years old, of both sexes ( 84 female gender), in order to implement a multifactorial program for the prevention of falls. This included analysis of the risk factors, incidence of falls and their consequences, through the evaluation of physical skills (muscle strength by dynamometry and balance using the platform of strength) and psychological (quality of life); and included also the use of social and health services. The authors concluded that this type of study assists in obtaining greater knowledge of risk factors of fall; allowing the development of programs that allow the identification of people with a propensity to fall in the third age. The aforementioned studies have used in their assessments, instruments with high technological content added, that, despite being extremely accurate and give high precision and reliability of the results, has restrictions in its use, because they are linked to research laboratories. Also, the fact that the technology employed requires specific qualification of personnel for handling and maintenance generates high financial cost and, therefore, are not accessible to many therapists. All of these factors were considered during the preparation of this study assessment protocol and were opted for the use of handy tools, with low cost finance, to provide convenience and reliability in carrying out evaluations; and that could be used routinely in everyday clinical therapeutic.

\subsection{Falls}

In relation to the number of falls, the results show that $40 \%$ of the sample reported having suffered at least one fall, in a period of 12 months. However, an important factor, and that can not be disregarded when it is questioned to an elderly population about falls, is the omission of fact. This can be justified by the oblivion due to functional decline of cognitive systems that occur because of advanced age, or because the fall is a sentinel event that potentially mark the beginning of body functional decline $[2,3,5]$. In relation to the genre, it was verified that the female group reports higher number of falls $(52.6 \%)$ than the male group $(14.3 \%)$. This result is consistent with several found in the literature, as the Perracini and Ramos [1], that occurred through two surveys in cohort study of elderly residents in Sao Paulo, showed that the higher prevalence of falls is in females. This fact can be justified due to the greater fragility, the higher prevalence of chronic diseases, the largest domestic activities link by placing these individuals in situations of higher risk of falling, and also due to the feminization of old age. This last factor was reported by Neri [9], when reporting about aging and quality of life in elderly woman. The author comments on the difference in relation to diseases that affect the genres. It is therefore verified the superiority of older women in relation to men. In relation to age, the female group FG2, above 70 years old, showed greater index of fall (61.8\%), followed by the male group MG2, i.e., regardless of genre, the older fall more. Despite this result, the risk factor, age, did not present relevance, or statistically significant correlation in any of the groups evaluated. Thus, they conclude that advancing age is no more, as advocated earlier, the predictor of the fall, despite the decline in physiological functional be progressive with age. A pool of factors should be evaluated in order to determine the predictors of fall, so that you can intervene before the occurrence of the event. So, is reinforced the need for protocols with multifunctional evaluations, but specific to each gender and age.

\subsection{Medication-Med}

$81.2 \%$ of the sample reports use of drugs, however, despite the high percentage, the frequency is two per day. In relation to the genre, the FG consumes more (87.7\%) than the MG and, regardless of sex, the older 
ingest more medications. On the correlation of medicines with the falls, the result was statistically significant only for the FG. The medication may be a factor to justify the result of high levels of registered fall within that group. This result is consistent with several studies described in the literature $[1,5,6]$. The medication has presented as a relevant risk factor when evaluating fall in a population of non institutionalized, of both sexes, aged 60 years old and above, requiring special investigation if the population is female, aged 70 years old above.

\subsection{Nutritional Status-BMI}

The BMI in the study did not show a significant correlation with the fall. It was verified a bipolarization of the nutritional profile, where the FG, overweight (45.6\%), and MG, low weight (46.4\%). This bipolarization was also verified by Tinoco [10] that assessed 183 elderly of both sexes aged between 60 and 90 years old and found a high prevalence of overweight female gender (19.5\%) and male, low weight (11.2\%). Saints and Sichieri [11] in a study that assessed 699 individuals of both sexes, over 60 years old, showed similar results, but only in relation to the female gender, which showed overweight. Acunã and Cruz [12] conducted a study on the nutritional situation of the brazilian population, researched the increasing prevalence of obesity in the elderly population, this being characterized as a global pandemic. The BMI gained prominence in the FG1, indicating that should be listed in protocols that evaluate a population with these characteristics.

\subsection{Circumference of the Calf-CC}

The results of this study demonstrate that the population has an adequate nutritional status, because $83.5 \%$ presented the $\mathrm{CC}>31 \mathrm{~cm}$. In relation to sex, the FG showed lower CC $(33 \mathrm{~cm})$ than MG $(37 \mathrm{~cm})$. This result can be justified due to muscle volume of individuals belonging to MG to be larger than the FG. In relation to age, regardless of gender, the elderly had lower circumferences of the calf, perhaps due to loss of muscle mass that happens with advancing age [13]. No statistically significant correlation was observed between this variable and fall by gender or age groups. However, analyzing the percentage of individuals who reported a fall $(40 \%)$, and considering the nutritional status of the population as being appropriate, it was verified that this risk factor can influence the fall. The $\mathrm{CC}$ can be considered as a relevant factor in female population aged above 70 years old, therefore, it is suggested the inclusion of this factor in the evaluation of protocols falls for this population. The study by Coelho [14] assessed the prevalence of malnutrition in 197 institutionalized elderly, with an average age of 79.3 years old, of both sexes, hospitalized in a geriatric unit in Belo Horizonte (MG, Brazil), and found that $57 \%$ of the population had the circumference $\leq 31 \mathrm{~cm}$. The authors highlight the need to use nutritional level indicators, because the elderly malnourished responds less to medicinal therapies, present the greatest risk of infection, and also the prejudice of functional independence and quality of life. This approach is consistent with the results of this study, where the MG1, who reported the lowest fall, presents greater circumference of the calf. On the other hand the FG2, reported highest fall and lower circumference of the calf and must appear on assessment protocols in female population aged 70 years old or more.

\subsection{Hand Grip Strength-HGS}

The results of this study demonstrate that individuals of the MG have higher average strength (31.6 kgF) than the FG (20.2 kgF). These results corroborate with those of Caporrino [15], which conducted a study population of hand grip strength, in upper limbs with Jamar ${ }^{\circledR}$ dynamometer, in 800 healthy individuals of both sexes. It was verified that the grip strength in men is significantly higher than in women. Also, Buchman [16] in their study found a difference between the sexes in relation to upper limb muscle strength. In the study, 234 men and 530 women, over 65 years old, were 
evaluated and found that muscle strength is higher in males than in females. The results in relation to age groups show that in FG, the decline of strength accompanies advancing age. However, in MG this fact is not checked, because the MG2 has the greatest strength (34.1 kgF) than MG1 (30.1 kgF). These results do not corroborate with Buchman [16], because they found that men's muscle strength declines with advancing age. They justify the fact due to declining levels of testosterone, and also because older men develop a wider variety of chronic diseases than women. They also found that men are stronger than women. Barbosa [17] also found that grip strength declines in more advanced age groups and is statistically lower in the female gender when compared to male. The results of this study demonstrate that there is correlation between the HGS and the fall in FG, corroborating with Stähelin [18]. They conducted a prospective study with 302 individuals, both sexes (40 men and 60 women), 70 years old above, with the goal of determining the risk factors of falls, and verified that the grip strength is an important risk factor to identify the elderly at risk of fall and to predict recurrent falls. The HGS has relevance in the elderly population presented in this study and also in the male population and group MG1. It is therefore suggested their inclusion in assessment protocols that use the theme of falling in elderly.

\subsection{Scales and Functional Tests}

\subsubsection{DGI (dynamic gait index)}

The population evaluated in this study showed average of 21.2 points, classified as normal. In relation to the genres, these showed similar results, male presented 21.7 points and female, 21.0 points. Results by age groups indicates that the FG2 has the lowest score (20.6 points), that is, that presents the lowest balance during walking in different contexts of functional tasks. It is important to emphasize that this group also presented the highest fall when compared to other groups. The correlation between this group and the falls was statistically significant, this result also observed when it was evaluated the female group. The DGI showed negative covariance in all groups of the study, indicating that the fall and the test vary in opposite directions, i.e., the higher the score on the test, the lower the risk of falling; and the smaller the test score, the greater the risk of falling. This test was presented as being a relevant risk factor when evaluating a sample of elderly, of both genders, not institutionalized, 60 years old above, especially when the group evaluated is male, aged 60 to 69 years old. In this context, it is suggested the inclusion of this test in evaluation protocols on falls in the elderly, in populations that present the above-mentioned characteristics.

\subsubsection{Timed Up and Go-TUG}

The average time achieved by the sample was $14.2 \mathrm{~s}$. In the MG the result was better than the FG (15.6 s). This result is not consistent with those of Bischoff [18]. They conducted a study, aiming at the establishment of a cutoff point for the normal mobility through the TUG in elderly women, living in community and institutionalized. 481 individuals were assessed (413 living in community and 78 institutionalized), aged between 65 and 85 years old, used the TUG, quantify the weight, height and BMI. It was verified that 92 women living in community, conducted the test in less than $15 \mathrm{~s}$, and 91 institutionalized women, took the test in less than $30 \mathrm{~s}$. Significant differences were observed in relation to the test execution time by sex and age groups, reinforcing the idea of establishing values by age groups. Thus, the affirmative corroborates with the study of Bohannon [19], who performed a meta-analysis, using 21 studies (1990 to 2005) on this subject. It has been found that the TUG could be categorized by age. Individuals aged between 60 and 69 years old showed average time of $8.1 \mathrm{~s}$ (interval of $[7.1 ; 9.0])$. For individuals from 70 to 79 years old, the average was $9.2 \mathrm{~s}$ (interval of $[8.2 ; 10.2]$ ) and, individuals from 80 to 99 years old, the average was $11.3 \mathrm{~s}$ (interval of $[10.0 ; 12.7])$. It was concluded that 
there is a need to standardize limits to evaluate and compare performance. The TUG performed statistically significant correlation with the fall and was presented as relevant risk factor in MG, MG1 and FG1, and should be included in protocols that evaluate especially these populations.

\subsubsection{Berg Balance Scale—BBS}

The sample mean was 48.7 points, above the cutoff point established by the scientific community as at risk of falling (45 points), therefore they are not at risk of falling. In relation to gender, they showed similar results: (1) FG: 48.9 points; (2) MG: 48.4 points. These results corroborate with Lajoie and Gallagher [20] who carried out a study to predict falls of elderly 125 (45 did not fall and 80 fell), with age between 65 and 99 years old, who lived in the community of Sudbury and belonged to YMCA (Young Men's Christian Association). Balance assessment were carried out (using a platform of strength), BBS and applied the questionnaire $\mathrm{ABC}$ (Activities-specific Balance Confidence). It was verified that individuals who have not fallen, presented greater balance, highest score in BBS and on ABC. The score of the BBS for those who reported no falls was 50.4 points and for those who fell, 36 points. It has been found that the tools used are significant contributors to predict the risk of falling in elderly and assists professionals to identify and monitor the potential for falls. The BBS parsed as a risk factor of falling in elderly, proved to be relevant, in the MG and MG2. Thus, it is suggested that this scale is included in assessment protocols falls in these populations.

\subsubsection{Functional Capacity-FC}

The results showed statistically significant correlation with fall only in FG that showed lower functional ability and greater reporting of falls. In contrast, the group MG1 showed lower difficulty to execute PDLA (performances of daily life activities), best cognitive index, greater functional capacity and the lowest fall. Similar results were reported by Rosa [21], they conducted a study to identify the determining factors of the functional capacity with individuals over 65 years old and concluded that the illiteracy, the pessimistic assessment of health to compare with their peers, and the mental health tracking, are fundamental factors for this identification, because it is related to the ability to deal with the work until older ages. Complementing these results, Franciulli [22] conducted a study for six months, with 14 elderly of both sexes aged over 80 years, and concluded that the functional capacity specifically in your motor size is an important marker for successful ageing, which translates into a better quality of life for the elderly. Thus, despite of this factor have not presented as relevant in any of the groups evaluated, it is suggested that it be investigated, because, it is known that the functional limitations are more governed by beliefs in own capacity, than by the level of physical disability; and are considered key elements in achieving, motivation and action about the events of daily life.

\subsubsection{Physical Self Efficacy Scale_-PES}

The sample mean in this factor was 67.4 points and indicates that individuals evaluated are confident in their ability to accomplish tasks using physical skills. In relation to gender, the male is more self confident and in relation to age, the MG1 obtained a greater prominence. Belief in self-efficacy according to Bandura [23] represent not only the skills and capabilities to respond to challenging situations, but also the confidence level to solve them interfere directly in this process. So, this can be the justification for the results obtained in the Group MG1, that showed lower drop index, higher life satisfaction (ESV) and higher perceived physical capacity and confidence in self-image. This result is consistent with those of $\mathrm{R}$ and Cardoso [24], who conducted a study whose objective was to show the relevance on the belief of self-efficacy, as protective mechanism or mediator in the adjustment of elderly disabled. It was verified that the elderly with greater sense of self-efficacy, sought active roles, in relation to the management of your health condition, they were less vulnerable to stress and depression, 
more persevering in the face of difficulties, and also in the development of their functional capabilities. Also, the study by Benites [25] assessed 642 individuals, between 16 and 81 years old, and found that the higher self-efficacy, best are the skills in daily life tasks executions. The PES in this study showed a statistically significant correlation with fall when considering the whole sample. In the analysis of covariance, it was verified that this was negative in all groups except the FG2. The negative covariances indicate that falls and the PES vary in opposite directions, i.e., the higher the physical self-efficacy, lower the number of falls and, the smaller the physical self-efficacy and the greater the number of falls. Despite the PES not having obtained featured in any of the age groups established in this study, it is important that it be evaluated, because as previously mentioned, the awareness of existing potential and physical capabilities, can collaborate effectively to participate in programs that benefit the physical health and psychological well-being. Thus, this factor can not be overlooked in evaluations about fall in elderly.

\subsubsection{Effectiveness of Falls Scale-FES-I-Brazil}

The fear of falling is defined as loss in confidence of the balance low self-efficacy in preventing falls, as well as an expression of anxiety or a concern over the falls. These feelings can limit the performance of the activity of the elderly on a day to day basis and, consequently, decrease quality of life [26].

In relation to gender, the women appeared to be more concerned and, above all, be the largest age group FG2, this group also showed greater fall indexes lower life satisfaction (Satisfaction with Life Scale-SLS) and less FC (functional capacity). These results corroborate with Musselman and Brouwer, Culham [27]. They carried out a cross-sectional study with 25 elderly, institutionalized not older than 65 years, of both sexes (16 women and 9 men), which was aim to verify that the physical conditions and the level of quality of life had correlation with the fear of falling. It has been verified that, individuals, which have been reported falling in the last year, had some feature restriction of activities when compared with those who did not fall. It was concluded that the decrease of strength and low perception of quality of life and poor health, had correlation with the fear of falling. Martin [28] searched for the association between the fear of falling and limitations of daily activities, in 713 females, through the application of the scale of fear of falling and SAFE (Survey of Activities and Fear of Falling in the Elderly), and verified that the fear of falling is responsible for the restriction of physical activities and also social. The FES-I-Brazil presented significant correlation with fall in FG and MG2. This is presented as relevant risk factor of fall and shall be recorded in protocols that evaluate individuals with age $\geq 60$ years old, of both sexes. Also, in protocols that evaluate a female population with the same features, and in a male population aged $\geq 70$ years old.

4.6.7 Satisfaction with Life Scale-SLS

The results related to life satisfaction demonstrate that $44.7 \%$ is very pleased with the life and none of the individuals reported being dissatisfied. The masculine gender is more pleased than the female and the FG2 are less satisfied than the other groups. Relating these results with the indices of fall, it was found that there is a close relationship between these factors, because the FG showed greater fall index, and the FG2 showed greater fall index and greater dissatisfaction with life. There was also negative covariance in all groups, that is, the more satisfied the individual is with his life, the lower the likelihood of a fall or vice versa. Thus, despite the SLS not having obtained highlight how relevant risk factor in groups, it should not be disregarded in the ratings on fall in elderly.

\section{Conclusions}

According to the results obtained, it is possible to affirm that for the evaluation of falling in elderly, you can not use only one single instrument search. It was also verified that the emotional status complements the information, and in this context, the individual can no 
longer be evaluated only about the physical-functional aspect, because it was evident the interference of these factors in falls. Variations in risk factors by gender and age, clearly demonstrate that the elderly can no longer be evaluated the same way and therefore can not be subjected to a standard intervention.

Thus, it is suggested that protocols are developed in a differentiated manner and on the basis of the risk factors identified, because these reflect the weaknesses of a specific population. This strategy is aim to ensure greater effectiveness, better targeting for evaluations and earliest interventions. Such specification levels can contribute to reducing the incidence of falls, and, consequently, providing improvement in the quality of life of a population expected to live increasingly.

\section{References}

[1] M.R. Perracini, L.R. Ramos, Factors associated with falls in a cohort of elderly residents in the community, Revista de Saúde Pública 36 (6) (2002) 709-716. (in Portuguese)

[2] D.C. Lojudice, Quedas de idosos institucionalizados: ocorrência e fatores associados, Dissertação de Mestrado, Medicina de Ribeirão Preto da Universidade, 2005. (in Portuguese)

[3] P. Kannus, H. Sievänene, M. Palvanen, T. Järvinen, J. Parkkari, Prevention of falls and consequent injuries in elderly people, Lancet 366 (2005) 1885-1893.

[4] E.C. Jorstad, K. Hauer, C. Becker, S.E. Lamb, Measuring the psychological outcomes of falling: a systematic review, Journal of the American Geriatrics Society 53 (3) (2005) 501-510.

[5] N.M. Sjösten, M. Salonja, M. Piirtola, T. Vahlberg, R. Isoaho, H. Hyttinen, et al., A multifactorial fall prevention programme in home-dwelling elderly people: A randomized-controlled trial, Public Health 121 (2007) 308-318.

[6] R.L. Junior, Participation in water gymnastics, self-efficacy beliefs and satisfaction with life in women from 50 to 70 years old, M.Sc. Dissertation, Universidade Estadual de Campinas, 2003. (in Portuguese)

[7] F.F. Camargos, Cross-cultural adaptation and evaluation of the psychometric properties of the Falls Efficacy Scale-International: an instrument to assess fear of falling in elderly, M.Sc. Dissertation, Universidade Federal de Minas Gerais, 2007. (in Portuguese)

[8] L.K. Boulgarides, S.M. McGinty, J.A. Willet, C.W. Barnes, Use of clinical and impairment-based tests to predict falls by community-dwelling older adults, Physical Therapy 83 (4) (2003) 328-339.

[9] A.L. Neri, Aging and quality of life in women, in: SBGG 2001, 2nd Congress Paulista de Geriatria e Gerontologia, Santos, São Paulo, 2001. (in Portuguese)

[10] A.L.A. Tinoco, L.F. Brito, M.S.L. Sant'anna, A.C. Mello, M.M.S. Silva, S.C.C. Frasncheschini, Overweight and obesity as measured by body mass index (BMI), waist circumference (CC) and waist hip ratio (WHR), of senior citizens of a city of Zona da Mata of Minas Gerais, Revista Brasileira de Geriatria e Gerontologia 9 (2) (2006) 46-51. (in Portuguese)

[11] D.M. Santos, R. Sichieri, Body mass index and anthropometric indicators of adiposity in the elderly, Revista de Saúde Publica 39 (2) (2005) 163-168.

[12] K. Acunã, T. Cruz, Assessment of nutritional status of Adults and the elderly and nutritional situation of the Brazilian population, Arquivos Brasileiros de Endocrinologia e Metabolismo 48 (3) (2004) 345-361.

[13] T.A.A. Silva, A.F. Junior, M.M. Pinheiro, V.L. Szejnfeld, Age-related Sarcopenia: etiological aspects and treatment options, Revista Brasileira de Reumatologia 46 (6) (2006) 391-397. (in Portuguese)

[14] A.K. Coelho, F.L. Rocha, M.A. Fausto, Prevalence of undernutrition in elderly patients hospitalized in a geriatric unit in Belo Horizonte, MG, Brazil, Nutrion 22 (10) (2006) 1005-1011.

[15] F.A. Caporrino, F. Faloppa, J.B.G. Santos, C. Réssio, F.H.C. Soares, L.R. Malachima, et al., Population study of the palmar prehension force with $\operatorname{Jamar}{ }^{\circledR}$ dynamometer, Revista Brasileira de Ortopedia 33 (2) (1998) 150-154. (in Portuguese)

[16] A.S. Buchman, R.S. Eilson, J.L. Bienias, D.A. Bennett, Gender differences in upper extremity motor performance of older persons, Geriatrics and Gerontology International 5 (2005) 59-65.

[17] A.R. Barbosa, J.M.P. Souza, M.L. Lebrão, R. Laurenti, M.F.N. Marucci, Functional limitation of Brazilian elderly by age and gender diferrences: data from SABE Survey, Caderno de Saúde Pública 21 (4) (2005) 177-185.

[18] H.A. Bischoff, H.B. Stähelin, A.U. Monsch, M.D. Iversen, A. Weyh, M. Dechen, et al., Identifying a cut-off point for normal mobility: a comparison of the timed "up and go" test in community-dwelling and institutionalised elderly women, Age and Aging 32 (2003) 315-320.

[19] R.W. Bohannon, Reference values for the timed up and go test: a descriptive meta-analysis, Journal of Geriatric Physical Therapy 29 (2) (2006) 64-68.

[20] Y. Lajoile, S.P. Gallagher, Predicting falls within the elderly community: comparison of postural sway, reaction time, the Berg balance scale and the Activities-specific Balance Confidence (ABC) scale for 
comparing fallers and non-fallers, Archieve Gerontology Geriatrics 38 (2004) 11-26.

[21] T.E.C. Rosa, M.H.A. Benício, M.R.D.O. Latorre, L.R. Ramos, Determining factors of functional capacity among elderly, Revista de Saúde Pública 37 (1) (2003) 40-48. (in Portuguese)

[22] S.E. Franciulli, N.A. Ricci, N.D. Lemos, R.C. Cordeiro, J.M. Gazzola, A modalidade de assistência Centro-Dia Geriátrico: efeitos funcionais em seis meses de acompanhamento multiprofissional, Ciência \& Saúde Coletiva 12 (2) (2007) 373-380. (in Portuguese)

[23] A. Bandura, Social cognitive theory: an agentic perspective, Annual Review of Psychology 52 (2001) 1-26.

[24] D.F. Rabelo, C.M. Cardoso, Auto-eficácia, doenças crônicas e incapacidade funcional na velhice, Psico-USF 12 (1) (2007) 75-81. (in Portuguese)
[25] D. Benites, S.M.C. Jacques, G. Gauer, W.B. Gomes, Percepção de auto-eficácia e auto relato de falhas de memória prospectiva e retrospective, Interação em Psicologia 10 (2) (2006) 207-215. (in Portuguese)

[26] L. Yardley, N. Beyer, K. Hauer, G. KEMPEN, P. Ziegler, C. Todd, Development and initial validation of the Falls Efficacy Scale-International (FES-I), Age and Aging 34 (2005) 614-619.

[27] B. Brouwer, K. Musselman, E. Culham, Physical function and health status among seniors with and without a fear of falling, Gerontology 50 (2004) 135-141.

[28] F.C. Martin, D. Hart, T. Spector, D.V. Doyle, D. Harari, Fear of falling limiting activity in young-old women is associated with reduced functional mobility rather than psychological factors, Age and Ageing 34 (2005) 281-287. 